\title{
Multiples and Parents of Multiples Prefer Same Arm Randomization of Siblings in Neonatal Trials
}

\author{
Janine Bernardo, BS, BA ${ }^{1,2}$, Amy Nowacki, PhD ${ }^{1,3}$, Richard Martin, MD ${ }^{2,4}$, Jonathan $\mathbf{M}$. \\ Fanaroff, MD, JD ${ }^{2,4}$, and Anna Maria Hibbs, MD, MSCE ${ }^{2,4}$ \\ ${ }^{1}$ Cleveland Clinic Lerner College of Medicine of Case Western Reserve University, Cleveland, $\mathrm{OH}$ \\ ${ }^{2}$ Case Western Reserve University School of Medicine, Cleveland, $\mathrm{OH}$ \\ ${ }^{3}$ Department of Quantitative Health Sciences, Cleveland Clinic Foundation, Cleveland, $\mathrm{OH}$ \\ ${ }^{4}$ Division of Neonatology, Rainbow Babies and Children's Hospital, Cleveland, $\mathrm{OH}$
}

\section{Abstract}

Objective-Although common among Neonatal Intensive Care Units, multiples births are randomized inconsistently within trials, which can impact enrollment, analytical approach, and trial outcomes. It is not known what randomization approach (same arm, different arm, independent randomization) is preferred by multiples and their families.

Study Design-Surveys distributed to parents of multiples and adult multiples addressed preferences on randomization by eliciting the most desired method and likelihood of enrolling twins for each randomization approach.

Result-Populations included 209 parents and 321 adult multiples. 78\% of parents and 59\% of multiples prefer same arm placement of multiples over other methods (both $p<0.001$ ), which also had highest likelihood of enrollment among both groups.

Conclusion-Parents of multiples and adult multiples prefer placement of multiples into same treatment arm in randomized trials, making such methodology a potential way to optimize consent rates while ethically approaching human subject research.

\section{Introduction}

Multiple gestation births are common among preterm and low birth weight infants and can make up a large minority, approximately $20 \%$ or more, of neonatal populations within Neonatal Intensive Care Units (NICU) ${ }^{1}$. Multiple births, of all orders, have seen an increase in the past several decades ${ }^{2}$, yet the issue of handling multiple gestations in neonatal research has lacked consistency in neonatal clinical trials ${ }^{1}$. Traditional statistical methods for clinical trials assume statistical independence of observations. However, multiples are

Corresponding author: Janine Bernardo, Cleveland Clinic Lerner College of Medicine of Case Western Reserve University, 9500 Euclid Avenue, NA21 Cleveland, OH 44195, Phone: 201-532-4023 / Fax: 216-844-3380, jpb111@ case.edu.

Authors have no conflicts of interest to declare.

Disclosures: The authors have no conflicts of interest to report.

Survey documents for parents of multiples and adult multiples are available at Journal of Perinatology's website. 
dependent observations, sharing genetic backgrounds, and similarly affected by prenatal, postnatal, and iatrogenic exposures. How multiples are randomized and analyzed in trials, therefore, can impact the analytic approach as well as the outcome of the trials; failure to account for the dependence of multiples, particularly when they compose a large proportion of the population, can result in inaccurate conclusions ${ }^{1,3-7}$. Little is known, however, about how multiples and their families would prefer to be included and randomized in neonatal trials.

There are three approaches in dealing with assignment of multiples: (1) assign patients to same treatment arm (same arm), (2) assign patients to opposite treatment arms (different arm), or (3) independently randomize them into treatment arms (independent randomization). Assigning patients to the same treatment arms has often been considered the most appealing in terms of compliance and parent preference, but such a method increases the clustering problem during statistical analyses, is computationally more difficult, and may require larger sample sizes ${ }^{6}$. However no actual data exists voicing the preferences of parents or adult multiples. Additionally, preferences are unknown in situations where not all of the multiples in a set qualify for study enrollment. Trial design that aligns with parental preferences is most satisfactory to families and may constitute a more ethical approach to conducting such human subjects research. The optimal approach to such issues has therefore not been established.

This study sought to examine parents' and adult multiples' preference on placement in randomized clinical trials through a survey questionnaire. Such a question is important as the answer will greatly impact future trial design and uncover the option most consistent with participant preference. We hypothesized that parents of multiples and adult multiples have a preferred randomization approach, which is to be placed together into the same treatment arm of randomized clinical trials and that randomization that forces twins into different treatment arms will be the least acceptable. We furthermore sought to assess the acceptability of enrolling one twin within a trial if only one twin is eligible.

\section{Methods}

Population

The population included adult parents of multiples and adult multiples who read English and have access to electronic distribution of survey links. Participation of neonates in clinical trials was not a requirement.

\section{Survey Design}

A 31 and 30 item survey was developed for parents of multiples and adult multiples, respectively. In addition to demographic questions, survey questions addressed parents' and multiples' preferences on randomization of twins. As we were interested in both identifying the most preferred method as well as quantifying the acceptability of each method, preference was assessed two ways: most preferred method of randomization (forcing the selection of one method among the three) and 5-point Likert scales given each method of randomization (rating each method individually). Such an approach was employed as it is 
quite possible for a parent to rate all three randomization methods as highly acceptable which would fail to indicate the most preferred method. The questions were asked under three scenarios relating to health of the child: when the health of the twins is unspecified, when a clinical scenario described the child as doing well and the risks of the trial minimal, and when a clinical scenario described the child as very sick and the child may benefit from participation. Likert scales also address participants' willingness to enroll into a trial in which one twin qualifies and the other does not, again asking when the health of the twin is unspecified, doing well, or very sick. The survey concludes with a final open-ended question asking for additional ideas regarding multiples in neonatal trials. Prior to distribution, the survey was screened by six lay persons, three physicians, and one statistician for readability and comprehension. The Flesch Kincaid Grade Level for the main text of the survey was calculated at a seventh grade reading level ${ }^{8}$. The description of randomization included a metaphor of a coin toss which determines the treatment arm a child may be placed into. The use of metaphors during consent to explain randomization in clinical trials, specifically a coin toss, is a commonly used method ${ }^{9}$.

Study data were collected and managed using REDCap electronic data capture tools hosted at University Hospitals. REDCap (Research Electronic Data Capture) is a secure, web-based application designed to support data capture for research studies.

\section{Survey Distribution}

The electronic surveys were distributed to parents of multiples and adult multiples through several venues. Organizations that distributed surveys include ResearchMatch.org, Multiples of America, TwinsDoctor.com, TwinsStuff.org, Preemiees Today, Northeast Ohio Mothers of Multiples, Ohio Federation of Mothers of Twins Clubs, Northern Dallas Mothers of Twins Club, Greater Rochester Mother of Twins Club and Case Daily (Case Western Reserve University's daily electronic newsletter). ResearchMatch is a free and secure registry which coordinates researchers with volunteers interested in participating in research studies. An optional lottery drawing for a $\$ 100$ Amazon giftcard was offered to all participants who completed the survey. Data collection occurred over a six-month period from November 2013-April 2014.

This study was approved by the University Hospitals Institutional Review Board.

\section{Statistical Approach}

Demographic characteristics of the participants were described using frequencies and percentages. A point estimate and $95 \%$ confidence interval summarize the proportion of parents and adult multiples having a randomization method preference. Identification of most preferred method utilized a goodness of fit chi square test against an equal distribution of one-third for each method. Wilcoxon rank sum tests examined whether the distribution of likeliness to enroll in a trial varied by randomization method within each health state and whether the distribution of likeness to enroll in a trial varied by health state within a given randomization method. Analysis of one twin enrollment preference was analyzed using a goodness of fit chi square test against an equal distribution of one-third for each rating. Wilcoxon rank sum test examined whether the distribution of likeliness to enroll one 
qualifying twin in a trial varied by health state. A level of 0.05 was used to determine statistical significance. JMP Pro 9.0 (Institute Inc., Cary, North Carolina, USA) was utilized for all statistical analyses.

Sample size justification was calculated based upon estimation of the proportion of parents having a randomization method preference. We hypothesized that $80 \%$ of survey participants would have a randomization method preference and an acceptable level of precision being $\pm 7.5 \%$ at the 0.05 significance level. Calculations indicated that at least 114 surveys would be required for both parents of multiples and adult multiples. Based upon first month response rates, we anticipated six months of data collection to accrue desired numbers; however, more surveys were collected towards the end of recruitment leading to a larger than anticipated sample size.

\section{Qualitative Description of Comments}

Qualitative responses to the open-ended question were coded with the following themes: guilt, responsibility, equality, health, risk, ease, study design, relief, stress, altruism, information, encouragement, distrust, and advice. "Guilt" was coded for responses which mentioned experiencing guilt from trial outcome based on decision. "Responsibility" was coded when responses mentioned the accountability of decision-making. "Health" and "risk" were coded for mention of health of the child or risk of intervention on decision-making. "Ease" means desiring the same treatment for twins because it would allow parents an easier time of administering a treatment or placebo. "Study design" referred to mention of clinical design features or structure, including the dependency of twin samples and randomization. "Relief" means being comforted by making certain decisions, particularly the independent randomization method. "Stress" was coded for responses which emphasized the stress faced by parents having children in the NICU. "Altruism" identified comments which highlighted participation in trials to benefit the greater good. Responses coded as "information" desired more information to be given in the clinical scenarios. "Encouragement" was coded for responses which provided support to the current or other research studies. "Distrust" and "advice" were coded for responses which mentioned doubts or recommendations for neonatal research, respectively.

\section{Results}

\section{Parent and Multiple Demographics}

A total of 209 parents and 321 adult multiples completed the surveys. Of note, 55\% of parents had children admitted to the NICU, while $29 \%$ of adult multiples were themselves admitted to the NICU. $19 \%$ of parents had children who were multiples enrolled in clinical trials and $9 \%$ of adult multiples were enrolled as children (Table 1).

\section{Randomization Preference}

A large majority of parents, $72 \%$ (95\% CI 66\% - 78\%) and adult multiples, $82 \%$ (95\% CI $78 \%-86 \%$ ) had a preference regarding the randomization approach of twins in neonatal trials for unspecified health status. Of those with a preference, same arm was the most preferred method among parent and adult multiple respondents in each health state; $78 \%$ of 
parents and 59\% of multiples preferred same arm placement when health was not specified state (chi square goodness of fit test, all $\mathrm{p}<0.001$, Figure 1). Among parents and multiples with NICU experience, similar results were observed; $83 \%$ of parents and $57 \%$ of adult multiples prefer same arm placement when health is not specified (data not shown).

\section{Influence of Randomization Approach on Trial Participation}

When asked to report likelihood of trial enrollment, the distribution of parental responses differed depending on the randomization method (Wilcoxon rank sum, $\mathrm{p}<0.001$, Table 2). When the randomization method was same arm and health unspecified $68 \%$ of parents were very or somewhat likely to enroll while only $28 \%$ of parents were very or somewhat likely to enroll when the randomization method was different arm and health unspecified. When the randomization method was independent randomization and health unspecified, more ambivalence is observed with $38 \%$ of parents very or somewhat likely to enroll and $42 \%$ very or somewhat unlikely to enroll. When we consider a specified health state, likeliness to enroll in trials utilizing the same arm and independent randomization, do not significantly differ (Wilcoxon rank sum, $\mathrm{p}=0.47, \mathrm{p}=0.08$ ). However, for different arm randomization, the proportion of parents very or somewhat likely to enroll increases statistically $(\mathrm{p}<0.001)$.

The distribution of adult multiple responses also differed depending on the randomization method (Wilcoxon rank sum, p <0.001, Table 2). Eighty-two percent of adult multiples were very or somewhat likely to enroll within the same arm randomization with health unspecified. When the randomization method was different arm and health unspecified, this declined to $51 \%$ very or somewhat likely to enroll. When the randomization method was independent randomization and health unspecified, 55\% of adult multiples were $42 \%$ very or somewhat unlikely to enroll. When we consider a specified health state, likeliness to enroll in trials utilizing the same arm did not significantly differ (Wilcoxon rank sum, $\mathrm{p}=0.92$ ), but did differ when among different arm and independent randomization (both $\mathrm{p}<0.001$ ).

\section{Likelihood of Participation with One Twin Enrollment}

When asked to report likelihood of trial enrollment when only one twin qualifies, the data indicate that parents have an opinion on enrollment regardless of health state (goodness of fit Chi square, all $\mathrm{p}<0.001$, Table 3 ). Nearly one half of parents (48\%) were very or somewhat willing to enroll one eligible twin when health is unspecified (Table 3). Perception of the infant being healthy or sick increased the proportion of parents very or somewhat likely to enroll by $4 \%$ and $12 \%$, respectively, but was not a statistically significant difference.

A larger percentage of multiples (73\%) were accepting of one twin enrollment when compared to parents (Table 3). Regardless of health scenario, adult multiples have a preference of enrollment (goodness of fit Chi square, all $p<0.001$, Table 3). An increase in acceptability was also noted among multiples when the one infant was described as being sick. Specification of health status resulted in a statistically significant change in acceptability of enrollment (Wilcoxon rank sum, p <0.004, Table 3). 


\section{Qualitative Responses}

Of the 209 parental responses, 80 (38\%) provided open-ended comments and amongst 312 multiples, 137 (44\%) did so. The most common thematic responses among parents included equality in treatment of twins (21\%), influence of health on decision (16\%), and distrust of research $(15 \%)$. Within multiple's responses, the most common themes were comments on study design (24\%), equality in treatment (22\%) and guilt related to decision making (14\%). The following are several representative comments which highlight these themes and others:

"I think it's so hard as a parent to make a decision that may give one twin a better chance at improvement/survival. How do you make that choice?"(Parent, same arm preference, theme: responsibility)

"I feel that if both were ill I wouldn't want them on the same drug for fear that if it didn't work, I could lose both babies. If they were on different drugs, hopefully one would work and I could at least save one of them."(Parent, different arm, theme: health)

"I think clinical trials of twins, especially identical, are a wonderful resource. I don't think the method affects if I'm more likely to participate although I would think being in separate groups would provide the most value."(Parent, different arm, themes: encouragement, study design)

"Even though twins have an equal chance of receiving drug $x$ or $y$ in all methods, it is difficult to see that. Parents try to stress equality more with multiples so researchers should try to emphasize the equal chances." (Adult multiple, same arm, theme: equality)

"I actually work as a lab tech for clinical trials, so I find this very interesting. On one hand I know why as a researcher you would want some of these scenarios, but as an identical twin, it would make it hard for me to deal with my twin getting different treatment than me... I have such a need for my sister to be healthy that it would devastate me if I found out later that she had a different treatment from me that either didn't help or made her health worse."(Adult multiple, same arm, themes: guilt, study design)

"Quite frankly, I'd rather let fate do the selecting for me than have to decide between those two options."(Adult multiple, independent randomization, theme: relief)

\section{Discussion}

This study explores the unanswered question of how parents of multiples and adult multiples prefer multiples to be randomized within neonatal trials. Results indicate that parents and adult multiples both have a preferred method of randomization which is to randomize multiple siblings into same treatment arms of clinical studies. Presentation of clinical scenarios, describing the child as either healthy or sick, appeared to increase likelihood or acceptability of participation. As mentioned in several open ended responses, parents may feel more altruistic when children are healthy and may contribute to advancement of 
knowledge, while perceiving the children as sick might make parents more willing to take the risk of enrolling their children for some potential benefit.

Each method of randomization has aspects which may be both appealing and unsatisfactory to family members, all of which were highlighted within open ended survey responses. Many parents and multiples equated the same arm placement with equal treatment and potentially increasing the chance for similar outcomes. This method may also avoid unblinding in cases in which families have direct exposure to the drug and placebo groups. Mandating separate grouping of twins may increase the chance that at least one twin may have the more effective treatment. This method also maximizes sample size in the setting of non-independence of twin pairs. Yet, survey respondents reflected that they may suffer psychological trauma if one twin has better/worse outcomes than the other if they were in separate arms. Allowing each twin's placement to be randomized independently treats the twins as if they were separate entities and removes the decision from the parents, somewhat decreasing the burden of decision making. We pose that while each option may be ethically equivalent if clinical equipoise and other fundamental principles are maintained, the potential psychological stress of separating the twins should be considered ${ }^{10,11}$.

In regards to one twin eligibility, approximately half of parents (48\%) would enroll only one eligible twin within studies, which was again influenced by the one infant being sick and potentially benefiting from treatment. A much greater percentage of adult multiples (73\%) found one twin enrollment acceptable. Some clinical trial designers may be hesitant to approach parents of multiples if only own infant is eligible for a trial for fear that parents would not be willing to enroll a single multiple. Yet, these results indicate that enrollment of a single twin is as likely to be viewed favorably as unfavorably, making pursuit of eligible multiples a worthwhile endeavor for infant's inclusion within the trial.

The current study emphasizes the importance of eliciting the perspective of both parents and adult multiples, as it may enhance the perspective of parents alone. Kodish et al. ${ }^{12}$ makes the case that informed pediatric consent for pediatric research may not be possible in the strict ethical and theoretical sense, but, rather, is composed of parental permission and, when possible, patient assent of an older child. In the case of neonatal research, informed consent relies strongly on the parental permission. It is possible that the combination of parental permission and assent of the older child may even be morally greater than informed consent of a competent adult ${ }^{12}$. Thus, we explored both perspectives.

The major strength of this study is that it addresses a critical methodologic issue in neonatal randomized controlled trials that has remained largely unaddressed within the community. It is anticipated that these results may improve awareness of the value of inclusion of multiples into clinical studies and, of great importance, influence future neonatal trial design. Of great importance, statistical methodology exists to incorporate the dependency of each randomization scheme, further supporting incorporation of the most acceptable method. Additionally, such research is not limited to a particular trial, disease, or treatment arm which expands the potential impact. Survey participants include a national representation of parents of multiples and adult multiples, additionally increasing external validity. Finally, and of much importance, is that the experience of a having sick children can be an extremely 
distressing one. Designing clinical trials in a way that is most satisfactory to a majority of parents should be implemented.

This study has the several limitations. Clinical trial practices, such as randomization, bias, and equipoise, are challenging principles for parents and family members to comprehend and accept and this study does not directly evaluate participant's understanding. Our study assumes that language typically used in consent forms is adequate to explain principle of randomization, yet acknowledges that in actual practice a large number of parents consenting to clinical trials may lack this understanding $9,13,14$. Our participants were well educated, with over three-quarters completing college, which makes the study less generalizable but suggests better comprehension. In addition, it would be most ideal to interview parents while in the NICU to ensure a thorough understanding of randomization and the most accurate picture of placement in neonatal trials. However, this may be difficult to achieve a high response rate and may be a burden on families acutely coping with the stress of ill infants. By exploring preference of parents with and without NICU experience, we found that preference did not differ significantly. In addition, while the survey presents different scenarios relating to disease severity of the infant, there are many specific details of neonatal clinical trials which we could not capture in this survey, such as the specific intervention of the trial, associated risks, etc. Future work may explore the nuances of such trial interventions.

In conclusion, our study has shown that the majority of parents of multiples and adult multiples have a preference on clinical trial randomization; they prefer same arm placement of multiples over different arm and independent randomization. Such results may inform future study design of neonatal clinical trials in such a way that will optimize participation of multiples and improve acceptability and consent. The population of multiple births is a significant one that cannot afford to be unnoticed within NICU trial populations.

\section{Acknowledgments}

We would like to thank all organizations who helped distribute the survey and all survey participants for their valued assistance and insight.

ResearchMatch is funded in part by the National Institutes of Health (NIH) Clinical and Translational Science Award (CTSA) program, grants UL1TR000445 and 1U54RR032646-01. The Clinical and Translational Science Collaborative (CTSC) grant supports (UL1TR000439) RedCap databases.

\section{References}

1. Hibbs AM, Black D, Palermo L, Cnaan A, Luan X, Truog WE, et al. Accounting for multiple births in neonatal and perinatal trials: systematic review and case study. J Pediatr. 2010; 156:202-8. [PubMed: 19969305]

2. Chauhan, SP. Twins: prevalence, problems, and preterm births. American Journal of Obstetrics and Gynecology. 2010; 203:305-315. [PubMed: 20728073]

3. Gates S, Brocklehurst P. How should randomised trials including multiple pregnancies be analysed? BJOG. 2004; 111:213-9. [PubMed: 14961881]

4. Heo M, Leon AC. Comparison of statistical methods for analysis of clustered binary observations. Statistics in Medicine. 2005; 24:911-923. [PubMed: 15558576] 
5. Yelland LN, Saltera AB, Ryan P, Makrides M. Analysis of binary outcomes from randomised trials including multiple births: when should clustering be taken into account? Paediatric and Perinatal Epidemiology. 2011; 25:283-297. [PubMed: 21470268]

6. Shaffer ML, Kunselman AR, Watterberg KL. Analysis of neonatal clinical trials with twin births. BMC Medical Research Methodology. 2009; 9:1-8. [PubMed: 19123933]

7. Marston L, Peacock JL, Yuc K, Brocklehurst P, Calvert SA, Greenough A, et al. Comparing methods of analysing datasets with small clusters: case studies using four paediatric datasets. Paediatric and Perinatal Epidemiology. 2009; 23:380-392. [PubMed: 19523085]

8. [Cited May 22, 2014] The Readability Test Tool [homepage on the Internet]. Available from: http:// read-able.com/

9. Kodish E, Eder M, Noll RB, Ruccione K, Lange B, Angiolillo A, et al. Communication of randomization in childhood leukemia trials. JAMA. 2004; 291:470-5. [PubMed: 14747504]

10. Emanuel EJ, Wendler D, Grady C. What makes clinical research ethical? JAMA. 2000; 283:27012711. [PubMed: 10819955]

11. Jonsen, A.; Siegler, M.; Winslade, W. Clinical Ethics: A Practical Approach to Ethical Decisions in Clinical Medicine. 7th. McGraw Hill; New York: 2002.

12. Kodish E. Informed consent for pediatric research: is it really possible? J Pediatr. 2003; 142:89-90. [PubMed: 12584521]

13. Snowdon C, Garrcia J, Elbourne D. Making sense of randomization; responses of parents of critically ill babies to random allocation of treatment in a clinical trial. Soc Sci Med. 1997; 45:1337-1355. [PubMed: 9351153]

14. Robinson EJ, Kerra C, Stevens A, Lilford R, Braunholtz D, Edwards S. Lay conceptions of the ethical and scientific justifications for randomallocation in clinical trials. Soc Sci Med. 2004; 58:811-824. [PubMed: 14672595] 

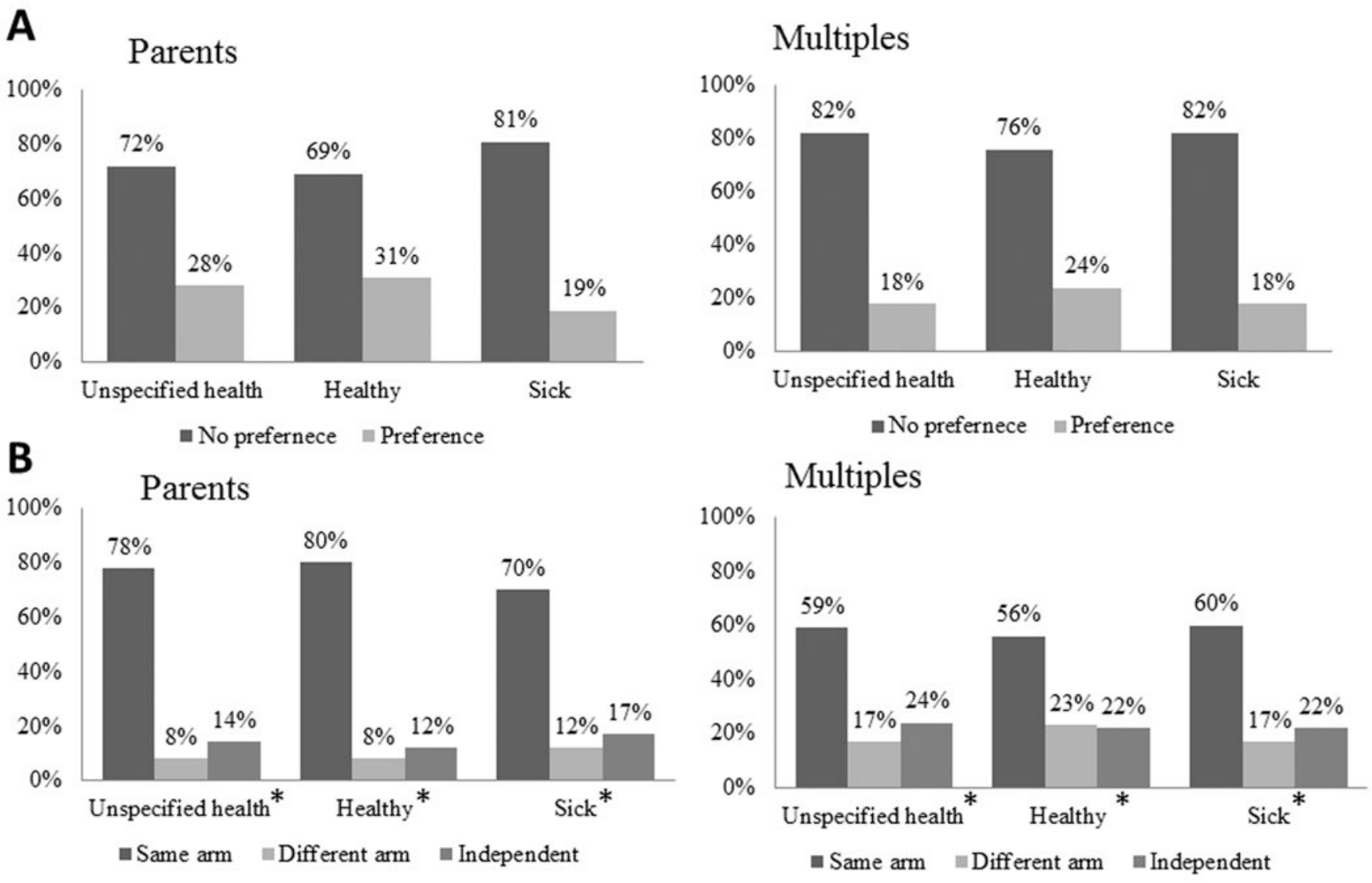

Figure 1. Parents and Multiples Preference on Randomization Approach

Parents of multiples and adult multiples who have a preference on how multiples are randomized within clinical trials based upon health status (A) and which method (same arm, different arm, independent randomization) is most preferred (B). In each of the six settings, same arm randomization was the most preferred (chi square goodness of fit test, all $\mathrm{p}<$ 0.001). 
Table 1

Demographic Characteristics of Parents and Multiples

\begin{tabular}{|c|c|c|}
\hline & Parents of Multiples & Adult Multiples \\
\hline & $\mathrm{N}=209$ & $\mathrm{~N}=321$ \\
\hline \multicolumn{3}{|l|}{ Age (years), n (\%) } \\
\hline $18-30$ & $20(10 \%)$ & $160(50 \%)$ \\
\hline $31-45$ & $141(67 \%)$ & $84(26 \%)$ \\
\hline $46-60$ & $39(19 \%)$ & $62(19 \%)$ \\
\hline$>61$ & $9(4 \%)$ & $15(5 \%)$ \\
\hline Gender (Male) & $5(2 \%)$ & $68(21 \%)$ \\
\hline \multicolumn{3}{|l|}{ Highest education completed } \\
\hline HS or GED & $29(14 \%)$ & $69(21 \%)$ \\
\hline College & $180(86 \%)$ & $251(78 \%)$ \\
\hline \multicolumn{3}{|l|}{ Type of multiple } \\
\hline Twins & $193(92 \%)$ & $312(97 \%)$ \\
\hline Identical, n (\%) & $42(22 \%)$ & $121(39 \%)$ \\
\hline Triplets & $14(7 \%)$ & $9(3 \%)$ \\
\hline \multicolumn{3}{|l|}{ Age of children who are multiples (years) } \\
\hline$\leq 5$ & $118(56 \%)$ & \\
\hline $6-10$ & $34(16 \%)$ & \\
\hline $11-20$ & $32(15 \%)$ & \\
\hline $21-35$ & $17(8 \%)$ & \\
\hline$>36-50$ & $8(3 \%)$ & \\
\hline Any admission to NICU & $114(55 \%)$ & $92(29 \%)$ \\
\hline \multicolumn{3}{|l|}{ Reasons for NICU admission } \\
\hline Prematurity & $94(82 \%)$ & $77(84 \%)$ \\
\hline Breathing problems & $59(52 \%)$ & $29(32 \%)$ \\
\hline Infection & $5(4 \%)$ & $3(3 \%)$ \\
\hline Feeding Problems & $40(35 \%)$ & $7(8 \%)$ \\
\hline Jaundice & $31(27 \%)$ & $7(8 \%)$ \\
\hline Nervous system condition & $3(3 \%)$ & $1(1 \%)$ \\
\hline Other & $14(12 \%)$ & $8(9 \%)$ \\
\hline I don't know & 0 & $4(4 \%)$ \\
\hline \multicolumn{3}{|l|}{ Participation in clinical trial as adult } \\
\hline Yes, I participated & $48(23 \%)$ & $130(40 \%)$ \\
\hline No, I declined participation & $5(2 \%)$ & $7(2 \%)$ \\
\hline No, I was never asked & $149(71 \%)$ & $179(56 \%)$ \\
\hline I don't know & $7(3 \%)$ & $5(2 \%)$ \\
\hline \multicolumn{3}{|c|}{ Children who are multiples or self participation in clinical trial as infant or child } \\
\hline Yes, participated & $39(19 \%)$ & $30(9 \%)$ \\
\hline No, declined participation for them & $6(3 \%)$ & $4(1 \%)$ \\
\hline No, never asked & $158(76 \%)$ & $231(72 \%)$ \\
\hline
\end{tabular}

J Perinatol. Author manuscript; available in PMC 2016 April 19. 


\begin{tabular}{lll}
\hline & Parents of Multiples & Adult Multiples \\
Unsure & $6(3 \%)$ & $56(17 \%)$ \\
\hline
\end{tabular}

HS: high school; GED: General Educational Development; NICU: Neonatal Intensive Care Unit 
Table 2

Influence of Randomization Approach on Trial Participation

\begin{tabular}{|c|c|c|c|c|}
\hline \multicolumn{5}{|c|}{ Parent Respondents } \\
\hline & Very or Somewhat likely to enroll & Neutral & Very or Somewhat unlikely to enroll & p-value \\
\hline \multicolumn{5}{|c|}{ Unspecified health } \\
\hline Same arm & $68 \%$ & $14 \%$ & $18 \%$ & \\
\hline Different arm & $28 \%$ & $15 \%$ & $57 \%$ & \multirow[t]{2}{*}{$\mathrm{p}<0.001$} \\
\hline Independent & $38 \%$ & $20 \%$ & $42 \%$ & \\
\hline \multicolumn{5}{|l|}{ Healthy } \\
\hline Same arm & $69 \%$ & $14 \%$ & $17 \%$ & \\
\hline Different arm & $41 \%$ & $19 \%$ & $40 \%$ & \multirow[t]{2}{*}{$\mathrm{p}<0.001$} \\
\hline Independent & $48 \%$ & $18 \%$ & $34 \%$ & \\
\hline \multicolumn{5}{|l|}{ Very sick } \\
\hline Same arm & $74 \%$ & $12 \%$ & $15 \%$ & \multirow{3}{*}{$\mathrm{p}<0.001$} \\
\hline Different arm & $44 \%$ & $13 \%$ & $43 \%$ & \\
\hline Independent & $47 \%$ & $16 \%$ & $37 \%$ & \\
\hline \multicolumn{5}{|c|}{ Comparisons across health state: same $\operatorname{arm}(\mathrm{p}=0.47)$, different $\operatorname{arm}(\mathrm{p}<0.001)$, independent $(\mathrm{p}=0.08)$} \\
\hline \multicolumn{5}{|c|}{ Adult Multiple Respondents } \\
\hline & Very or Somewhat acceptable & Neutral & Very or Somewhat unacceptable & p-value \\
\hline \multicolumn{5}{|c|}{ Unspecified health } \\
\hline Same arm & $82 \%$ & $12 \%$ & $7 \%$ & \multirow{3}{*}{$\mathrm{p}<0.001$} \\
\hline Different arm & $51 \%$ & $17 \%$ & $32 \%$ & \\
\hline Independent & $55 \% \%$ & $22 \%$ & $23 \%$ & \\
\hline \multicolumn{5}{|l|}{ Healthy } \\
\hline Same arm & $81 \%$ & $11 \%$ & $8 \%$ & \multirow{3}{*}{$\mathrm{p}<0.001$} \\
\hline Different arm & $66 \%$ & $15 \%$ & $19 \%$ & \\
\hline Independent & $68 \%$ & $18 \%$ & $14 \%$ & \\
\hline \multicolumn{5}{|l|}{ Very sick } \\
\hline Same arm & $80 \%$ & $12 \%$ & $7 \%$ & \multirow{3}{*}{$\mathrm{p}<0.001$} \\
\hline Different arm & $52 \%$ & $16 \%$ & $32 \%$ & \\
\hline Independent & $57 \%$ & $19 \%$ & $24 \%$ & \\
\hline Comparisons acr & ealth state: same arm $(p=0.92)$, dif & rent arm & $<0.001)$, independent $(\mathrm{p}<0.001)$ & \\
\hline
\end{tabular}

J Perinatol. Author manuscript; available in PMC 2016 April 19. 
Table 3

Likelihood of Participation with One Twin Enrollment

\begin{tabular}{|c|c|c|c|c|}
\hline \multicolumn{5}{|c|}{ Parent Respondents } \\
\hline & Very or Somewhat likely to enroll & Neutral & Very or Somewhat unlikely to enroll & p-value \\
\hline Unspecified & $48 \%$ & $18 \%$ & $34 \%$ & \\
\hline Healthy & $52 \%$ & $19 \%$ & $29 \%$ & $\mathrm{p}=0.17$ \\
\hline Very sick & $60 \%$ & $8 \%$ & $32 \%$ & \\
\hline \multicolumn{5}{|c|}{ Chi square goodness of fit: unspecified ( $p<0.001$ ), healthy ( $<<0.001$ ), very sick ( $<<0.001$ ) } \\
\hline \multicolumn{5}{|c|}{ Adult Multiple Respondents } \\
\hline & Very or Somewhat acceptable & Neutral & Very or Somewhat unacceptable & $p$-value \\
\hline Unspecified & $73 \%$ & $11 \%$ & $17 \%$ & \\
\hline Healthy & $68 \%$ & $16 \%$ & $16 \%$ & $\mathrm{p}=0.004$ \\
\hline Very sick & $80 \%$ & $10 \%$ & $10 \%$ & \\
\hline
\end{tabular}

